\title{
Single-stage repair of arch aneurysms with a long elephant trunk: Medium-term follow-up of thromboexcluded aneurysms
}

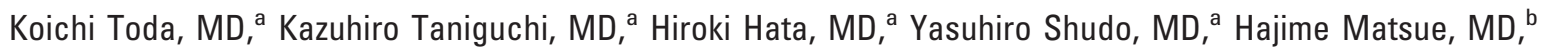
Satoru Kuki, MD, ${ }^{c}$ and Yoshiki Sawa, $M^{b}{ }^{b}$

From the Department of Cardiovascular

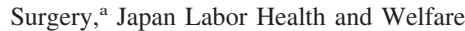
Organization Osaka Rosai Hospital, Sakai, Japan; the Department of Cardiovascular Surgery, ${ }^{\mathrm{b}}$ Osaka University Graduate School of Medicine, Suita, Japan; and the Department of Cardiovascular Surgery, ${ }^{\mathrm{c}}$ Takarazuka Municipal Hospital, Takarazuka, Japan.

Received for publication Nov 21, 2006; revisions received Feb 20, 2007; accepted for publication Feb 23, 2007

Address for reprints: Koichi Toda, MD, 1179-3, Nagasone-cho, Kita-ku, Sakai, Osaka, Japan (E-mail: ktoda2002@yahoo. co.jp).

J Thorac Cardiovasc Surg 2007;134:47-52

$0022-5223 / \$ 32.00$

Copyright (C) 2007 by The American Association for Thoracic Surgery

doi:10.1016/j.jtcvs.2007.02.030
Objectives: The purpose of this study was to investigate the medium-term results of arch aneurysms repaired by total arch replacement with a long elephant trunk and to evaluate whether this technique requires a subsequent distal anastomosis at the descending aorta when complete aneurysmal thrombosis is achieved around a long elephant trunk.

Methods: From June 1999 through May 2005, 32 consecutive patients with arch aneurysms underwent total arch replacement with a long elephant trunk anastomosed at the base of the innominate artery. Postoperatively, aneurysm size was evaluated by means of serial computed tomographic scanning.

Results: None of the patients experienced a new stroke, although there was $1(3 \%)$ hospital mortality. Computed tomographic scanning demonstrated complete thrombosis of the aneurysm in $29(91 \%)$ patients within 1 month after surgical intervention, and 3 patients with incomplete thrombosis of the aneurysm underwent a subsequent distal anastomosis in the descending aorta. The 3-year survival rate was $87 \%$, with no aneurysm rupture or sudden death. In the 29 patients who showed complete thrombosis of the aneurysm surrounding a long elephant trunk, serial computed tomographic scanning revealed a significant reduction in the size of the thrombosed aneurysm ( $81 \%$ at 1 year and $76 \%$ at 2 years after surgical intervention), and there was no case that showed expansion of the aneurysm.

Conclusions: Arch aneurysms were repaired safely by means of total arch replacement with a long elephant trunk, and successful shrinkage of the arch aneurysm suggests that this technique does not require subsequent distal anastomosis and could turn the 2-stage elephant trunk procedure into a single-stage repair when complete aneurysmal thrombosis is achieved.

A $\mathrm{n}$ aortic arch aneurysm extending to the descending aorta presents a surgical challenge, and the optimal technique remains controversial. A single-stage procedure with large incisions carries a substantial mortality and morbidity, ${ }^{1}$ and thus a 2-stage approach with an elephant trunk anastomosed in the distal aortic arch has been widely used since its introduction by Borst and colleagues ${ }^{2}$ as a less-invasive procedure. However, the cumulative risk of two major procedures and the additional risk of rupture between the two procedures have been demonstrated. ${ }^{3}$ We first introduced total arch replacement (TAR) with a long elephant trunk (LET) anastomosed at the base of the innominate artery to reduce stress on the elephant trunk anastomosis by placing the anastomosis in the less-dilated and less-diseased segment of the aorta. ${ }^{4}$ In our original series all patients underwent the second-stage elephant trunk procedures with a mean interval of 8.8 days, and there was no interval death or hospital mortality after either procedure. In this series we found that the aneurysms in most cases were completely thrombosed around the LET. We 


\author{
Abbreviations and Acronyms \\ $\mathrm{CT}=$ computed tomography \\ LET $=$ long elephant trunk \\ $\mathrm{TAR}=$ total arch replacement
}

speculated that aneurysmal expansion could be prevented and a second-stage procedure might not be necessary when the aneurysmal sac is completely thrombosed around the LET. The present study was conducted to further investigate operative and medium-term follow-up results in consecutive patients for whom an arch aneurysm was repaired by means of TAR with an LET.

\section{Materials and Methods \\ Patients}

Between June 1999 and May 2005, 41 patients with arch aneurysms underwent TAR with an LET. Among them, 32 consecutive patients whose arch aneurysms did not extend beyond the level of the carina were investigated in this study after approval was obtained from the institutional review board and informed consent was provided by each patient. The baseline patient clinical characteristics, including associated comorbidity, are shown in Table 1. Of the 32 patients, 2 had a poor left ventricular ejection fraction of less than $40 \%$, and 1 was receiving hemodialysis because of chronic renal failure. Four patients had significant coronary artery disease, which required a preoperative percutaneous coronary intervention in 2 and concomitant coronary artery bypass grafting in 2 , whereas 5 patients required aortic root replacement with a composite graft, 2 required aortic valve replacement, and 1 required aortic valve replacement and mitral valve replacement concomitantly.

\section{Surgical Procedures}

The present surgical technique of TAR with an LET anastomosed at the base of the innominate artery was previously reported. ${ }^{4}$ Briefly, the ascending aorta and arch vessels were minimally dissected by using a median sternotomy. Patients were started on cardiopulmonary bypass with the arterial return to an 8-mm Dacron graft (Gelweave, Vascutek, Germany) anastomosed to the

\section{TABLE 1. Preoperative characteristics of the patients}

$\begin{array}{lc}\text { Age }(\mathrm{y}) & 69 \pm 8(45-83) \\ \text { Male sex } & 23 / 32(72 \%) \\ \text { Cerebral infarction } & 6 / 32(19 \%) \\ \text { CAD } & 4 / 32(13 \%) \\ \text { Valvular disease } & 8 / 32(25 \%) \\ \text { AAA } & 6 / 32(19 \%) \\ \text { Size of aneurysm (mm) } & 59 \pm 10(45-83) \\ \text { Type of aneurysm } & \\ \text { Saccular } & 12 / 32(38 \%) \\ \text { Fusiform } & 14 / 32(44 \%) \\ \text { Type A dissection } & 6 / 32(19 \%)\end{array}$

$C A D$, Coronary artery disease; $A A A$, abdominal aortic aneurysm.

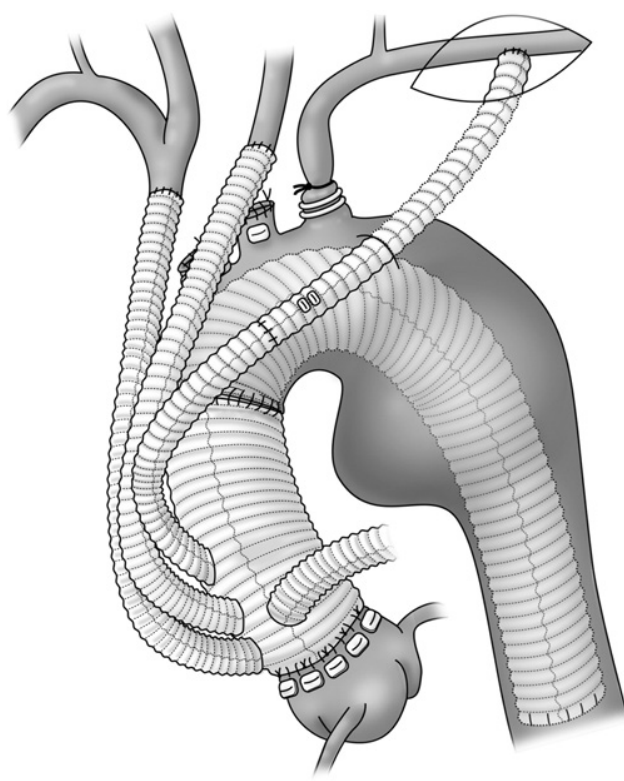

Figure 1. Total arch replacement with a long elephant trunk anastomosed at the base of the innominate artery.

right axillary artery and venous drainage from the bicaval cannula and then cooled down to $25^{\circ} \mathrm{C}$. While cooling the patient, the heart was arrested with antegrade and retrograde cold blood cardioplegia, after which a 4-branched arch graft (Hemashield Gold woven double velour vascular graft; Boston Scientific, Natick, Mass) was sutured into the sinotubular junction. The size of the 4-branched arch graft was determined based on the size of the descending aorta in preoperative computed tomographic (CT) scans. When the patient had been cooled to $25^{\circ} \mathrm{C}$, systemic perfusion was stopped, and selective cerebral perfusion was then instituted with an additional cannula in the left common carotid artery and the 8-mm Dacron graft (Gelweave) anastomosed to the left axillary artery. The ascending aorta was transected at the base of the innominate artery, and the LET was placed into the aortic arch and descending aorta by pulling the LET with a catching catheter (Amplatz GooseNeck Snare; Microvena, White Bear Lake, Minn) introduced from the femoral artery. We determined the length of the LET by measuring the greater curvature of the lumen of the aorta from the base of the innominate artery to the end of the aneurysm with a CT angiogram obtained before the operation. A distal anastomosis was then performed at the base of the innominate artery between the 4-branched arch graft and the LET tube graft incorporating the distal ascending aorta. After antegrade distal perfusion was resumed from the side branch of the graft, the arch vessels were divided and anastomosed to the branches of the graft. (Figure 1).

\section{Follow-up and Data Analysis}

All patients underwent a CT scan within 1 month after surgical intervention. Those patients who demonstrated complete thrombosis of the arch aneurysm on the CT scan were discharged and followed on an outpatient basis, during which serial CT scans were performed every 6 months in the first year and every 12 months 
thereafter. The remaining patients who did not demonstrate complete thrombosis of the aneurysm on CT scans required a secondstage procedure, which has been described previously. ${ }^{5}$ Briefly, the descending aorta was exposed through an anterolateral left thoracotomy, and after the descending aorta was clamped during normothermic femorofemoral venoarterial bypass, the LET was pulled out and the distal end was anastomosed to the descending aorta. In these patients preoperative variables were investigated by means of review of the clinical records, and postoperative variables were investigated by means of review of the outpatient records and a cross-sectional follow-up examination conducted in September 2006 by telephone interview.

Data were analyzed with StatView software (version 5; SAS, Cary, NC). Values are expressed as the mean \pm standard deviation. The Fisher exact test was used for categoric variables, and the Mann-Whitney $U$ test or Wilcoxon signed-rank test was used to compare continuous variables. Survival curves were generated by using Kaplan-Meier methods.

\section{Results}

The mean duration values for aortic crossclamp time, open distal time, and antegrade selective cerebral perfusion time were $86 \pm 51,23 \pm 8$, and $99 \pm 22$ minutes, respectively. There were no operative deaths or mortalities within 30 days of the operation, although there was $1(3 \%)$ hospital mortality. That patient had a long history of hemodialysis and a history of cerebral aneurysm, had mediastinitis postoperatively, and eventually died of multiorgan failure caused by sepsis 3 months after the operation. None of the patients had a new stroke, recurrent nerve palsy, or phrenic nerve palsy, whereas $1(3 \%)$ patient had permanent paraplegia caused by a spinal cord infarction at the level of Th1 and 1 required a tracheostomy for prolonged ventilation. Postoperative enhanced CT scans revealed patent branched grafts in all cases. CT scans also demonstrated complete thrombosis of the aneurysm in $29(91 \%)$ patients within 1 month after the operation (Figure 2, A), whereas the remaining 3 patients did not show complete thrombosis of the aneurysms (Figure 2, $C$ ) and underwent a subsequent distal anastomosis in the descending aorta through a left thoracotomy. The mean duration between the first and second procedures for those 3 patients was $18 \pm 14$ days (range, 7-34 days). Of the patients who required a second-stage procedure, the operative and cardiopulmonary bypass times were $136 \pm 15$ and $33 \pm 4$ minutes, respectively, and none required blood transfusion in the second procedure. CT scans 1 month after the second procedure demonstrated complete thromboexclusion of the aneurysm in those 3 patients.

The length and diameter of the LET in all patients were $16 \pm 3 \mathrm{~cm}$ (range, $10-20 \mathrm{~cm}$ ) and $25 \pm 2 \mathrm{~mm}$ (range, 22-28 mm), respectively. The distal end of the LET, which was marked with a metal clip, was confirmed by means of CT scans between the Th5 and Th10 levels $(8.0 \pm 1.3)$. The geometry of the aorta was compared between the patients
(A)
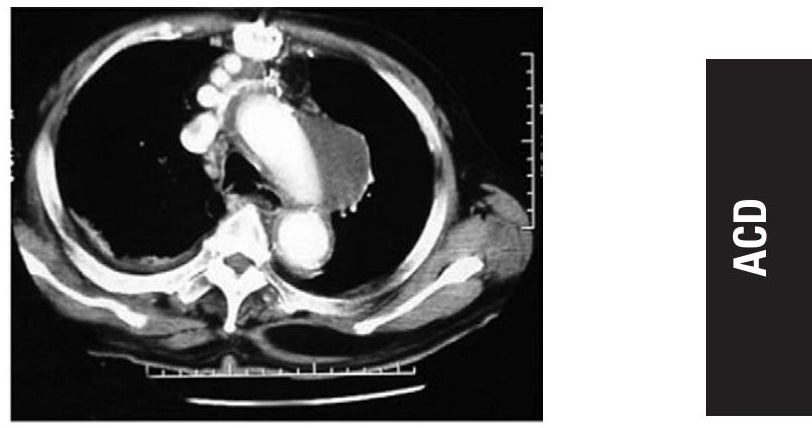

(B)

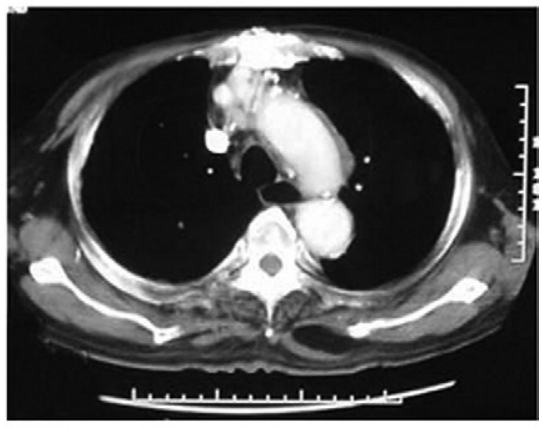

(C)

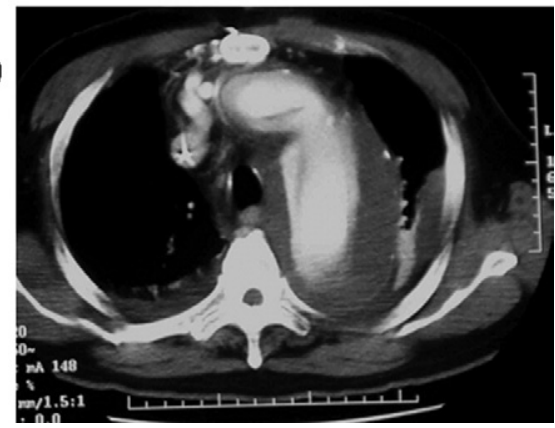

Figure 2. Contrast computed tomographic scans of the thromboexcluded (A) and nonthromboexcluded (C) arch aneurysms at 1 month after total arch replacement with a long elephant trunk. The thromboexcluded arch aneurysm (A) showed shrinkage at 1 year after total arch replacement with a long elephant trunk (B).

who did not demonstrate complete thrombosis of the aneurysm and subsequently required the second procedure and those who demonstrated complete thrombosis of the aneurysm (Table 2). The maximum diameter of the aneurysm and the diameter of the descending aorta at the level of the carina were significantly less in the patients who had complete thrombosis of the aneurysm, and therefore the LET had a significant contact zone against the wall of the descending aorta. All 28 patients who had an aneurysm with a maximum diameter of less than $7 \mathrm{~cm}$ demonstrated complete thrombosis of the aneurysm by the LET, whereas only 1 among 4 patients with an aneurysm larger than $7 \mathrm{~cm}$ demonstrated complete thromboexclusion by the LET 
Table 2. Comparison of the size of the aneurysm and descending aorta between thrombosed and nonthrombosed arch aneurysms

\begin{tabular}{|c|c|c|c|}
\hline & $\begin{array}{c}\text { Thrombosed } \\
\text { aneurysm } \\
(n=29)\end{array}$ & $\begin{array}{l}\text { Nonthrombosed } \\
\text { aneurysm } \\
(n=3)\end{array}$ & $P$ value \\
\hline Size of aneurysm (mm) & $57 \pm 8$ & $77 \pm 6$ & .012 \\
\hline $\begin{array}{l}\text { Size of descending } \\
\text { aorta }(\mathrm{mm})\end{array}$ & $29 \pm 2$ & $33 \pm 2$ & .011 \\
\hline Contact zone (mm) & $52 \pm 16$ & 0 & .0034 \\
\hline
\end{tabular}

Size of aneurysm, Maximum diameter of the arch aneurysm measured on preoperative computed tomographic scan; size of descending aorta, diameter of the descending aorta measured on preoperative computed tomographic scan at the level of the carina; contact zone, longitudinal length of the contact zone between the long elephant trunk and wall of the descending aorta measured on computed tomographic scan 1 month after surgical intervention.

(100\% vs $25 \%, P=.0008)$. As for the type of aneurysm, all 12 saccular and all 6 dissected aneurysms were completely thrombosed around the LET, whereas 3 of 14 fusiform aneurysms demonstrated incomplete aneurysmal thrombosis $(P=.1191)$.

All patients but 1 who died in the hospital were followed for more than 1 year, and the mean follow-up period was $33 \pm 18$ months. The 3-year survival rate, as determined with a Kaplan-Meier curve, was $87 \%$ (Figure 3). During the follow-up period, no aneurysm rupture or sudden death occurred, and there was no need for a second-stage procedure in the 29 patients who showed complete thrombosis of the aneurysm within 1 month after the operation. There were late deaths in 4 patients, 2 of whom died of pneumonia at 1 year after the operation, whereas 1 died of a cerebellum hemorrhage 14 months after the operation and 1 died of hepatic failure 4 years after the operation.

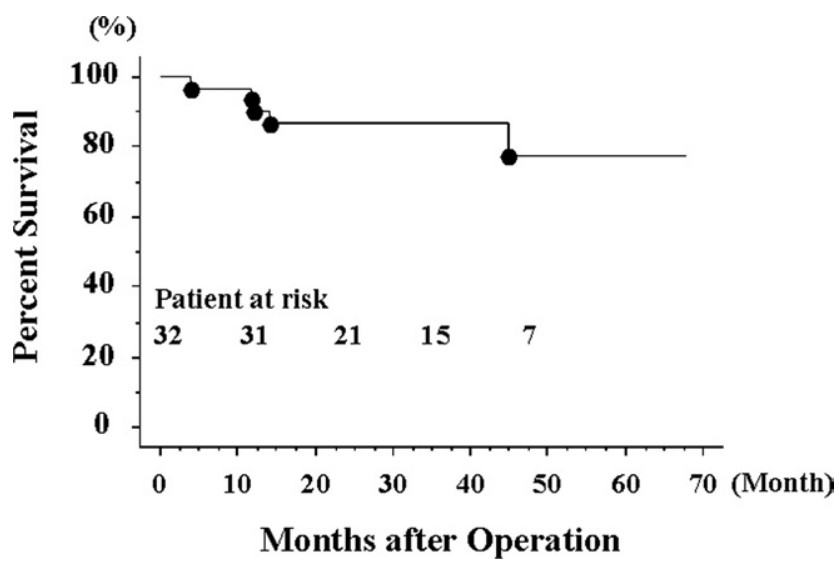

Figure 3. Kaplan-Meier survival curves after total arch replacement with a long elephant trunk.

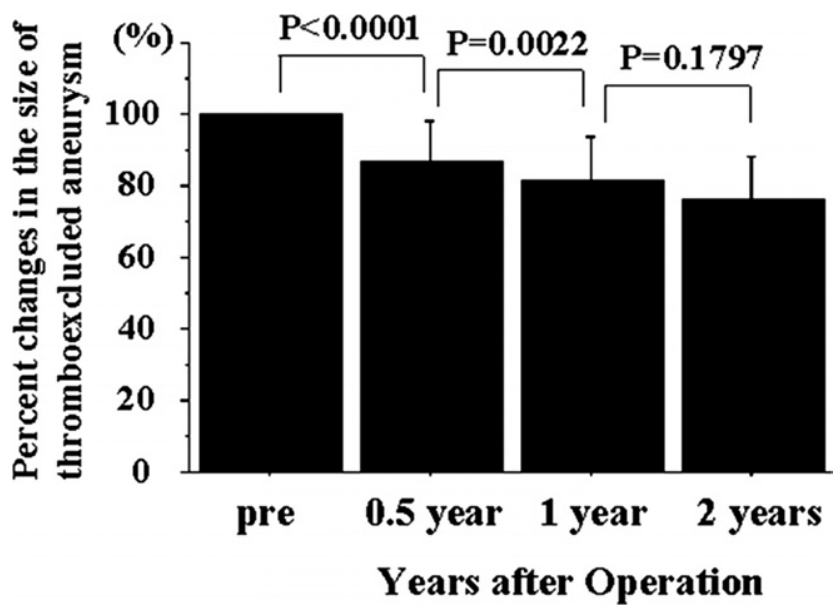

Figure 4. Percentage changes in the size of the thromboexcluded aneurysms at 6 months, 1 year, and 2 years after total arch replacement with a long elephant trunk.

The aneurysm size was measured by using serial CT scans. Figure $2, B$, shows shrinkage of a representative thrombosed arch aneurysm (Figure 2, $A$ ) at 1 year after TAR with an LET. None of the completely thrombosed aneurysms showed expansion, and the thrombosed aneurysms surrounding the LET shrank more than $5 \mathrm{~mm}$ in 23 (82\%) cases and more than $10 \mathrm{~mm}$ in $14(50 \%)$ cases at 1 year after TAR with an LET. Figure 4 shows significant reductions in size of the thrombosed aneurysms to $87 \% \pm 12 \%$ at 6 months, $81 \% \pm 12 \%$ at 1 year, and $76 \% \pm 12 \%$ at 2 years after TAR with an LET.

\section{Discussion}

The stent graft repair of thoracic aneurysms has been widely performed since the Stanford group demonstrated that a complete thromboexclusion of an aneurysm by a stent graft could prevent the expansion and eventual rupture of a thoracic aneurysm. ${ }^{6}$ However, the fate of a thromboexcluded aneurysm surrounding a tube graft without a stent is unknown, although the thromboexclusion of a proximal descending aneurysm by an elephant trunk has been demonstrated by Borst and colleagues. ${ }^{2}$ The present study showed that aneurysmal thrombosis surrounding the LET was completed in the majority of patients with arch aneurysms (91\%) within 1 month after performing a TAR with an LET, and aneurysmal expansion and rupture were prevented in all cases with an aneurysm thromboexcluded by the LET. In addition, the maximum diameter of the thromboexcluded aneurysms was significantly reduced by a mean of $76 \%$ at 2 years after surgical intervention. Although the follow-up period might not be long enough (mean, 33 months) to draw a firm conclusion, our medium-term results suggest that an arch aneurysm without extension beyond the 
level of the carina can be repaired by using an LET anastomosed at the base of the innominate artery and that our technique does not require a subsequent distal anastomosis through a left thoracotomy when the aneurysm is thromboexcluded by the LET.

A second-stage procedure was necessary in $3(9 \%)$ patients who did not show complete thrombosis of the aneurysmal sac surrounding the LET on postoperative CT scans. Preoperative CT scans revealed that all 28 patients who had an aneurysm with a maximum diameter of less than $7 \mathrm{~cm}$ demonstrated complete thrombosis of the aneurysm by the LET, whereas only 1 among 4 patients with an aneurysm larger than $7 \mathrm{~cm}$ demonstrated complete thromboexclusion by the LET ( $100 \%$ vs $25 \%, P=.0008)$. This suggests that complete thromboexclusion of an arch aneurysm larger than $7 \mathrm{~cm}$ might be less likely with TAR with an LET alone, and an additional procedure is necessary to complete the aneurysmal thrombosis around the LET. Although we could apply a second-stage procedure as an additional procedure for those 3 patients without mortality or blood transfusion, transcatheter placement of an endovascular stent graft, which would anchor the floating LET to the descending aorta, ${ }^{7}$ might be an alternative to complete the aneurysmal thrombosis around the LET. As long as those additional procedures are possible, our technique could be applied for patients with an arch aneurysm. On the other hand, in case of patients with ruptured aneurysms, a single-stage TAR through a median sternotomy alone ${ }^{8}$ or a bilateral anterior thoracotomy ${ }^{9}$ would be required because those patients are too unstable to undergo an additional procedure.

Concerning patients with more extensive involvement of the descending aorta, we would apply a rapid 2-stage procedure, as we have previously reported. ${ }^{4}$ We think that our LET technique is more advantageous than a standard LET technique, especially in patients with a dilated descending aorta, because the interval death caused by anastomotic rupture was avoided by placing the anastomosis in the less-dilated and less-diseased segment of the distal ascending aorta. ${ }^{4}$

Karck and associates ${ }^{10}$ demonstrated that a stent graft combined with a conventional vascular prosthesis could turn a 2-stage elephant trunk procedure into a single-stage repair through a sternotomy. Their method, the so-called frozen elephant truck technique, was used in 22 patients with arch aneurysms, and thromboexclusion was obtained in $20(91 \%)$ patients. However, the aneurysm was ruptured in 1 patient because a rigid stent penetrated the aneurysm during insertion of the stent graft. Stent-related complication, such as an aneurysmal rupture caused by graft perforation from mechanical stress on the stent, was also reported even 5 years after the frozen elephant trunk procedure. ${ }^{11}$ Using the present method, none of the patients had an aortic dissection or a rupture during the operation or postopera- tively. Because we did not use a rigid stent inside the graft, the aortic wall was not injured, even in cases of acute aortic dissection. Although no stent-related complications occurred, kinking of the graft could be a problem. To prevent such kinking, we introduced the graft into the descending aorta by pulling it straight into the aorta with a catching wire placed from the femoral artery rather than by pushing it into the aorta. With this technique, none of our 32 cases demonstrated a kinking of the LET, and this technique also prevented injury to the aortic wall when the graft was introduced without causing a large amount of shear stress on the aortic wall.

One $(3 \%)$ of our 32 patients experienced paraplegia caused by a spinal cord infarction at the level of Th1. Because the stent graft and the LET are introduced in the descending aorta, which might have a major feeding artery to the anterior spinal artery, spinal cord ischemia is another issue to be considered in the frozen elephant trunk procedure, as well as the LET technique. The mechanism of spinal cord ischemia appears to be multifactorial and remains ill-defined. Miyairi and coworkers ${ }^{12}$ demonstrated that a frozen elephant trunk procedure for aortic arch aneurysm was associated with an increased risk of paraplegia (21.1\%) and that a spinal cord ischemic time of more than 60 minutes was a risk factor for paraplegia. We performed open distal anastomoses within 45 minutes in all of the present cases, with an average time of $23 \pm 8$ minutes. In addition to reducing the risk of anastomotic rupture, anastomosing the LET at the distal ascending aorta yields a secure and rapid anastomosis and is expected to contribute to a decrease in spinal cord ischemic time and risk of paraplegia.

Thromboembolism from severe atherosclerosis of the distal landing zone was also reported as a cause of spinal cord ischemia. ${ }^{13}$ To prevent a debris-related embolism during placement of the LET, we washed out the debris from the LET by perfusing the descending aorta through a small arterial cannula placed in the femoral artery before completing the distal anastomosis.

Extensive deployment of the stented elephant trunk was associated with increased risk of spinal cord injury. ${ }^{14}$ In the present case of spinal cord infarction, postoperative CT scans showed the distal end of the LET at the level of Th8. We always attempt to not place the graft beyond the level of Th8 based on the assumption that the critical intercostal arteries exist below that level. If those arteries can be identified preoperatively, their location should be taken into consideration before determining the length of the LET. Recently, we found that the end of the graft could be seen with an intraoperative transesophageal echocardiogram. This technology could be applied to confirm the end of the graft intraoperatively, thus avoiding occlusion of the critical intercostal arteries by an excessively long LET. 
In summary, arch aneurysms without extension beyond the level of the carina were repaired safely by using TAR with an LET, and successful shrinkage of the arch aneurysm suggests that this technique does not require a subsequent distal anastomosis and could turn the 2-stage elephant trunk procedure into a single-stage repair when complete aneurysmal thrombosis is achieved around the LET.

\section{References}

1. Minale C, Splittgerber FH, Reifschneider HJ. Replacement of the entire thoracic aorta in a single stage. Ann Thorac Surg. 1994;57: 850-5.

2. Borst HG, Frank G, Schaps D. Treatment of extensive aortic aneurysms by a new multiple-stage approach. $J$ Thorac Cardiovasc Surg. 1988;95:11-3.

3. Estrera AL, Miller CC 3rd, Porat EE, Huynh TT, Winnerkvist A, Safi HJ. Staged repair of extensive aortic aneurysms. Ann Thorac Surg. 2002;74(suppl):S1803-5.

4. Kuki S, Taniguchi K, Masai T, Endo S. A novel modification of elephant trunk technique using a single four-branched arch graft for extensive thoracic aortic aneurysm. Eur J Cardiothorac Surg. 2000; 18:246-8.

5. Kuki S, Taniguchi K, Masai T, Yokota T, Yoshida K, Yamamoto K, et al. An alternative approach using long elephant trunk for extensive aortic aneurysm: elephant trunk anastomosis at the base of the innominate artery. Circulation. 2002;106(suppl 1):I253-8.
6. Mitchell RS, Dake MD, Semba CP, Fogarty TJ, Zarins CK, Liddell $\mathrm{RP}$, et al. Endovascular stent-graft repair of thoracic aortic aneurysms. J Thorac Cardiovasc Surg. 1996;111:1054-62.

7. Greenberg RK, Haddad F, Svensson L, O'Neill S, Walker E, Lyden SP, et al. Hybrid approaches to thoracic aortic aneurysms: the role of endovascular elephant trunk completion. Circulation. 2005;112:2619-26.

8. Kazui T, Washiyama N, Muhammad BA, Terada H, Yamashita K, Takinami M. Improved results of atherosclerotic arch aneurysm operations with a refined technique. J Thorac Cardiovasc Surg. 2001;121: 491-9.

9. Kouchoukos NT, Mauney MC, Masetti P, Castner CF. Single-stage repair of extensive thoracic aortic aneurysms: experience with the arch-first technique and bilateral anterior thoracotomy. J Thorac Cardiovasc Surg. 2004;128:669-76.

10. Karck M, Chavan A, Khaladj N, Friedrich H, Hagl C, Haverich A. The frozen elephant trunk technique for the treatment of extensive thoracic aortic aneurysms: operative results and follow-up. Eur J Cardiothorac Surg. 2005;28:286-90.

11. Toyama M, Usui A, Yoshikawa M, Ueda Y. Thoracic aneurysm rupture due to graft perforation after endovascular stent-grafting via median sternotomy. Eur J Cardiothorac Surg. 2005;27:162-4.

12. Miyairi T, Kotsuka Y, Ezure M, Ono M, Morota T, Kubota H, et al. Open stent-grafting for aortic arch aneurysm is associated with increased risk of paraplegia. Ann Thorac Surg. 2002;74:83-9.

13. Miyairi T, Kotsuka Y, Morota T, Kubota H, Shibata K, Ikeda Y, et al. Paraplegia after open surgery using endovascular stent graft for aortic arch aneurysm. J Thorac Cardiovasc Surg. 2001;122:1240-3.

14. Flores J, Kunihara T, Shiiya N, Yoshimoto K, Matsuzaki K, Yasuda K. Extensive deployment of the stented elephant trunk is associated with an increased risk of spinal cord injury. J Thorac Cardiovasc Surg. 2006;131:336-42. 